\title{
On inequalities of subgroups and the structure of finite groups
}

Jinbao Li ${ }^{1}$ and Fengyan Xie ${ }^{2^{*}}$

\author{
"Correspondence: \\ kfxiefengyan@163.com \\ ${ }^{2}$ Mathematics and Information \\ Engineering Department, \\ Humanistic Management College of \\ Anyang Normal University, Anyang, \\ 455000, P.R. China \\ Full list of author information is \\ available at the end of the article
}

\begin{abstract}
Let $G$ be a group and $H$ be a subgroup of $G$. We say that $H$ is weakly $\Phi$-supplemented in $G$ if $G$ has a subgroup $T$ such that $H T=G$ and $H \cap T \leq \Phi(H)$, where $\Phi(H)$ denotes the Frattini subgroup of $H$. In this paper, properties of this new kind of inequalities of subgroups are investigated and new characterizations of nilpotency and supersolubility of finite groups in terms of the new inequalities are obtained.
\end{abstract}

MSC: 20D10; 20D15; 20D20

Keywords: finite groups; weakly $\Phi$-supplemented subgroups; nilpotent groups; supersoluble groups

\section{Introduction}

All groups in this paper are finite.

Let $G$ be a group and $H$ be a subgroup of $G$. $H$ is said to be complemented in $G$ if $G$ has a subgroup $K$ such that $G=H K$ and $H \cap K=1$. A lot of information about the structure of finite groups can be obtained under the assumption that some families of subgroups are complemented (cf., e.g., [1-4]). For example, a classical result of Hall is about the solubility of a group $G$ satisfying that every Sylow subgroup of $G$ is complemented in $G$ [3]. A subgroup $H$ of a group $G$ is said to be supplemented in $G$ if $G$ has a subgroup $T$ such that $G=H T$. It is clear that every subgroup of a group $G$ is supplemented in $G$ and every complemented subgroup is also a supplemented subgroup. However, supplemented subgroups may not be complemented. Based on this investigation, we introduce the following new inequalities of subgroups related closely to supplementarity of subgroups.

Definition 1.1 Let $G$ be a group and $H$ be a subgroup of $G$. $H$ is said to be weakly $\Phi$-supplemented in $G$ if $G$ has a subgroup $T$ such that $G=H T$ and $H \cap T \leq \Phi(H)$, where $\Phi(H)$ is the Frattini subgroup of $H$.

By the definition, a complemented subgroup is still a weakly $\Phi$-supplemented subgroup. However, the converse does not hold.

Example 1.2 Let $G=Q_{8}$, the quaternion group of order 8, and let $H$ be a subgroup of $G$ of order 4. Then $H$ is weakly $\Phi$-supplemented in $G$ but not complemented in $G$.

This example shows that the class of all weakly $\Phi$-supplemented subgroups is wider than the class of all complemented subgroups. Thus, a question arises naturally:

() $2013 \mathrm{Li}$ and Xie; licensee Springer. This is an Open Access article distributed under the terms of the Creative Commons Attribution License (http://creativecommons.org/licenses/by/2.0), which permits unrestricted use, distribution, and reproduction in any medium, provided the original work is properly cited. 
Can we characterize the structure of finite groups in terms of the weakly $\Phi$-supplemented subgroups?

In this paper, we try to study this question and characterize the structure of groups by this new kind of inequalities of subgroups. In Section 3, we present a new criterion for the nilpotency of finite groups. In Section 4, a characterization of supersolubility of groups is given under the assumption that cyclic subgroups of order prime or 4 are weakly $\Phi$-supplemented.

The notation and terminology in this paper are standard and the reader is referred to [5] if necessary.

\section{Preliminaries}

In this section, we give some lemmas which will be useful in the sequel.

Lemma 2.1 Let G be a group. Suppose that $H$ is a weakly $\Phi$-supplemented subgroup of G.

(1) If $H \leq M \leq G$, then $H$ is weakly $\Phi$-supplemented in $M$.

(2) Suppose that $N \unlhd G$ and $N \leq H$. Then $H / N$ is weakly $\Phi$-supplemented in $G / N$.

(3) If $E$ is a normal subgroup of $G$ and $(|H|,|E|)=1$, then $H E / E$ is weakly $\Phi$-supplemented in $G / E$.

Proof (1) If $H$ is weakly $\Phi$-supplemented in $G$, there exists a subgroup $T$ in $G$ such that $H T=G$ and $H \cap T \leq \Phi(H)$. Since $H \leq M, H(T \cap M)=H T \cap M=M$ and $H \cap(T \cap M) \leq$ $(H \cap T) \cap M \leq \Phi(H) \cap M=\Phi(H)$, hence $H$ is weakly $\Phi$-supplemented in $M$.

(2) Since $H$ is weakly $\Phi$-supplemented in $G$, there exists a subgroup $T$ such that $H T=G$ and $H \cap T \leq \Phi(H)$. Then it is easy to see that $(H / N)(T N / N)=G / N$ and $(H / N) \cap(T N / N)=$ $(H \cap T) N / N \leq \Phi(H) N / N \leq \Phi(H / N)$. Therefore, $H / N$ is weakly $\Phi$-supplemented in $G / N$.

(3) Assume that $H$ is weakly $\Phi$-supplemented in $G$, and let $T$ be a subgroup of $G$ such that

$$
H T=G \text { and } H \cap T \leq \Phi(H) .
$$

Then $(H E / E)(T E / E)=(H T) E / E=G / E$. Since $(|H|,|E|)=1$,

$$
(|H E \cap T: H E \cap T \cap H|,|H E \cap T: H E \cap T \cap E|)=1 .
$$

Hence, we have that

$$
H E \cap T=(H E \cap T \cap H)(H E \cap T \cap E)=(H \cap T)(E \cap T) .
$$

It follows that

$$
(H E / E) \cap(T E / E)=(H E \cap T) E / E=(H \cap T) E / E \leq \Phi(H) E / E \leq \Phi(H E / E) .
$$

This shows that $H E / E$ is weakly $\Phi$-supplemented in $G / E$.

Recall that a group $G$ is called quasinilpotent if given any chief factor $H / K$ of $G$, every automorphism of $H / K$ induced by an element of $G$ is inner; the generalized Fitting subgroup $F^{*}(G)$ of $G$ is the product of all normal quasinilpotent subgroups of $G$. The following 
well-known facts about the generalized Fitting subgroup of a group $G$ will be used in our proofs (see [6, Chapter X] and [7, Lemma 4]).

Lemma 2.2 Let $G$ be a group.

(1) If $G$ is quasinilpotent and $N$ is a normal subgroup of $G$, then $N$ and $G / N$ are quasinilpotent.

(2) If $N$ is a normal subgroup of $G$, then $F^{*}(N)=N \cap F^{*}(G)$.

(3) $F(G) \leq F^{*}(G)=F^{*}\left(F^{*}(G)\right)$. If $F^{*}(G)$ is soluble, then $F^{*}(G)=F(G)$.

(4) Let $p$ be a prime and $P$ be a normal p-subgroup of $G$. Then $F^{*}(G / \Phi(P))=F^{*}(G) / \Phi(P)$. If $P$ is contained in $Z(G)$, then $F^{*}(G / P)=F^{*}(G) / P$.

\section{New characterizations of nilpotency}

Theorem 3.1 Let $G$ be a group with a normal subgroup $N$ such that $G / N$ is p-nilpotent. Suppose that every minimal subgroup of $N$ of order $p$ is contained in $Z(G)$, and every cyclic subgroup of $N$ with order 4 (if $p=2)$ is weakly $\Phi$-supplemented in $G$. Then $G$ is p-nilpotent.

Proof Suppose that the assertion is not true, and let $G$ be a counterexample of minimal order. Then:

(1) $G$ is a minimal non-nilpotent group and $G=P \rtimes Q$, where $Q$ is a Sylow $q$-subgroup of $G, P / \Phi(P)$ is a chief factor of $G$ and $\exp (P)=p$ or $\exp (P)=4$.

Let $L$ be a proper subgroup of $G$. Because $L / L \cap N \simeq L N / N \leq G / N$ and $G / N$ is $p$ nilpotent, $L / L \cap N$ is $p$-nilpotent. By the hypothesis and Lemma 2.1, every cyclic subgroup of $L \cap N$ with order 4 (if $p=2$ ) is weakly $\Phi$-supplemented in $L$. Since every minimal subgroup of $N$ of order $p$ is contained in $Z(G)$ and $Z(G) \cap L \leq Z(L)$, every minimal subgroup of $L \cap N$ of order $p$ is contained in $Z(L)$. Therefore $L$ satisfies the hypothesis. Hence, by the choice of $G, L$ is $p$-nilpotent. It follows that $G$ is a minimal non-p-nilpotent group. Then, by [8, Chapter IV, Theorem 5.4] and [5, Theorem 3.4.11], $G$ has a normal Sylow $p$-subgroup $P$ satisfying that $G=P \rtimes Q$, where $Q$ is a Sylow $q$-subgroup of $G, P / \Phi(P)$ is a chief factor of $G$ and $\exp (P)=p$ or $\exp (P)=4$.

(2) There exists an element with order 4 of $P$.

It is easy to see that $P$ is contained in $N$. Assume that (2) is false. Then $\exp (P)=p$ by (1). By the hypothesis, $P$ is contained in $Z(G)$. Therefore $G$ is nilpotent. This contradiction shows that (2) holds.

(3) The final contradiction.

Let $x \in P$ and $|x|=4$. Then $\langle x\rangle$ is weakly $\Phi$-supplemented in $G$. Thus there exists a subgroup $T$ of $G$ such that $\langle x\rangle T=G$ and $\langle x\rangle \cap T \leq \Phi(\langle x\rangle)=\left\langle x^{2}\right\rangle$. Since $P / \Phi(P) \cap T \Phi(P) / \Phi(P)$ is normal in $G / \Phi(P)$, we have $P \cap T \Phi(P)=P$ or $\Phi(P)$. If $P \cap T \Phi(P)=P$, then $P \leq T$ and therefore $\langle x\rangle \cap T=\langle x\rangle$, a contradiction. Hence $P \cap T \Phi(P)=\Phi(P)$ and so $P=P \cap\langle x\rangle T=$ $\langle x\rangle(P \cap T)=\langle x\rangle$. It follows from [8, Chapter IV, Theorem 2.8] that $G$ is nilpotent. This contradiction completes the proof.

Theorem 3.2 Let $G$ be a group with a normal subgroup $N$ such that $G / N$ is nilpotent. Suppose that every minimal subgroup of $F^{*}(N)$ is contained in $Z(G)$ and that every cyclic subgroup of $F^{*}(N)$ with order 4 is weakly $\Phi$-supplemented in $G$. Then $G$ is nilpotent.

Proof Suppose that the statement is not true, and let $G$ be a counterexample of minimal order. 
Let $M$ be a proper normal subgroup of $G$. We argue that $M$ satisfies the hypothesis. Since $M / M \cap N \simeq M N / N \leq G / N, M / M \cap N$ is nilpotent. By Lemma 2.2, $F^{*}(M \cap N) \leq F^{*}(N)$. Hence every minimal subgroup of $F^{*}(M \cap N)$ is contained $Z(M)$, and every cyclic subgroup of $F^{*}(M \cap N)$ of order 4 is weakly $\Phi$-supplemented in $M$ by Lemma 2.1. Therefore $M$ satisfies the hypothesis and so it is nilpotent by the minimality of $G$. Furthermore, we have that $F(G)$ is the unique maximal normal subgroup of $G$ and $G / F(G)$ is a chief factor of $G$. In view of Theorem 3.1, we also have $N=G=G^{\mathfrak{N}}$, where $G^{\mathfrak{N}}$ denotes the smallest normal subgroup of $G$ such that $G / G^{\mathfrak{N}}$ is nilpotent. Since $Z_{\infty}(G) \cap G^{\mathfrak{N}} \leq Z\left(G^{\mathfrak{N}}\right)[5$, Corollary 3.2.9], we have $Z_{\infty}(G)=Z(G)$. Let $F^{*}(G)=F$, let $p$ be the smallest prime dividing the order of $F$, and let $P$ be the Sylow $p$-subgroup of $F$. Then $F$ is a proper normal subgroup of $G$ by Theorem 3.1 and $P$ is normal in $G$. Let $Q$ be an arbitrary Sylow $q$-subgroup of $G$ with $q \neq p$, a prime. By Lemma 2.1 and Theorem 3.1, $P Q$ is $p$-nilpotent and so $Q$ is contained in $C_{G}(P)$. This implies that $O^{p}(G) \leq C_{G}(P)$. Hence $G=C_{G}(P)$ since $G=G^{\mathfrak{N}}$. Then $P \leq Z(G)$. By Lemma 2.2, $F^{*}(G / P)=F^{*}(G) / P$. Obviously, 2 does not divide the order of $F^{*}(G / P)$. By Lemma 2.1, $G / P$ fulfils the condition and so it is nilpotent by the choice of $G$, which shows that $G$ is nilpotent, a finial contradiction completing the proof.

\section{New characterizations of supersolubility}

Lemma 4.1 Let $p$ be the smallest prime dividing the order of a group $G$, and let $P$ be a Sylow $p$-subgroup of $G$. Then $G$ is p-nilpotent if and only if every cyclic subgroup of $P$ of order prime or 4 (if $P$ is a non-abelian 2-group) not having a supersoluble supplement in $G$ is weakly $\Phi$-supplemented in $G$.

Proof The necessity part is obvious. We prove the sufficiency. Suppose it is false. Then $G$ is non- $p$-nilpotent and so $G$ contains a minimal non- $p$-nilpotent subgroup $A$. Then $A$ is a minimal non-nilpotent group and possesses the following properties: (1) $A=\left[A_{p}\right] A_{q}$, where $A_{p}$ is the Sylow $p$-subgroup of $A, A_{q}$ is the Sylow $q$-subgroup of $A$ and $A_{p}$ is the smallest normal subgroup of $A$ such that $A / A_{p}$ is nilpotent; (2) $A_{p} / \Phi\left(A_{p}\right)$ is a chief factor of $A$; (3) $\exp \left(A_{p}\right)=p$ or 4 . Without loss of generality, we suppose that $A_{p} \leq P$. It is easy to see from Lemma 2.1 that every cyclic subgroup of $A$ of order $p$ or 4 (if $p=2$ ) not having a supersoluble supplement in $A$ is weakly $\Phi$-supplemented in $A$. Let $x$ be an element of $A_{p}$ such that $x \notin \Phi\left(A_{p}\right)$ and $H=\langle x\rangle$. Then $H$ is of order $p$ or 4 . Furthermore, one can suppose that $H$ does not have any supersoluble supplement in $A$ because if every cyclic subgroup of $A$ of order $p$ or 4 has a supersoluble supplement in $A$, then $A$ is nilpotent. Then, by the hypothesis, $H$ is weakly $\Phi$-supplemented in $G$ and so $A$ has a subgroup $T$ such that $A=H T$ and $H \cap T \leq \Phi(H)$. Since $A_{p} / \Phi\left(A_{p}\right) \cap T \Phi\left(A_{p}\right) / \Phi\left(A_{p}\right)$ is normal in $A / \Phi\left(A_{p}\right)$, $A_{p} \cap T \Phi\left(A_{p}\right)=A_{p}$ or $\Phi\left(A_{p}\right)$ by (2). If the former occurs, then $G=T$ and $H \cap T=H$, a contradiction. Suppose the latter holds, then $A_{p}=H$, which implies that $A$ is nilpotent, a final contradiction completing the proof.

Lemma 4.2 Let $P$ be a non-trivial normal p-subgroup of $G$, where $p$ is a prime. If $\exp (P)=p$ and every minimal subgroup of $G$ not having a supersoluble supplement in $G$ is weakly $\Phi$-supplemented in $G$, then every chieffactor of $G$ below $P$ is cyclic.

Proof Denote $\Phi(P)$ by $L$. Consider the factor group $P / L$. We verify that $P / L$ is a normal subgroup of $G / L$ satisfying the hypothesis. Clearly, $\exp (P / L)=p$. Let $H / L$ be a minimal 
subgroup of $P / L$. Then $H / L=\langle x\rangle L / L$ for some $x \in H \backslash L$. Then, by the hypothesis, $|x|=p$ and so $\langle x\rangle$ either has a supersoluble supplement in $G$ or is weakly $\Phi$-supplemented in $G$. If $\langle x\rangle$ has a supersoluble supplement $T$ in $G$, then $T L / L$ is a supersoluble supplement of $H / L$ in $G / L$. If $\langle x\rangle$ is weakly $\Phi$-supplemented in $G$, then $G$ has a subgroup $T$ such that $G=\langle x\rangle T$ and $\langle x\rangle \cap T \leq \Phi(\langle x\rangle)=1$. Therefore

$$
G / L=(\langle x\rangle L / L)(T L / L)=(H / L)(T L / L) \quad \text { and } \quad H / L \cap T L / L \leq \Phi(H / L)=1,
$$

implying that $H / L$ is weakly $\Phi$-supplemented in $G / L$. Hence $P / L$ satisfies the hypothesis and consequently, by induction, every chief factor of $G / L$ below $P / L$ is cyclic provided that $L \neq 1$. Thus, every chief factor of $G$ below $P$ is cyclic. Now suppose that $L=\Phi(P)=1$. Then $P$ is elementary abelian of exponent $p$. Let $N$ be a minimal subgroup of $P$. Suppose that $N$ has a supersoluble supplement $T$ in $G$. If $N \leq T, G=T$ is supersoluble and the conclusion follows. If $N \cap T=1$, then $P=P \cap N T=N(P \cap T)$. Since $P$ is abelian, $P \cap T$ is normal in $G$ and so every chief factor of $G$ below $P \cap T$ is cyclic by induction. It follows that the result holds. If $N$ is weakly $\Phi$-supplemented in $G$, then $G$ has a subgroup $T$ such that $N \cap T \leq \Phi(N)=1$. As above, we have that $P \cap T$ is normal in $G$ and every chief factor of $G$ below $P \cap T$ is cyclic. Since $P=N(P \cap T)$, every chief factor of $G$ below $P$ is cyclic. Thus, the proof is complete.

Theorem 4.3 Let $G$ be a group. Then $G$ is supersoluble if and only if $G$ has a normal subgroup $E$ such that for each non-cyclic Sylow subgroup $P$ of E, every cyclic subgroup of $P$ of order prime or 4 (if $P$ is a non-abelian 2-group) without any supersoluble supplement in $G$ is weakly $\Phi$-supplemented in $G$.

Proof The necessity is clear and we only need to prove the sufficiency. We proceed the proof by induction. By Lemmas 2.1 and 4.1, $E$ is $p$-nilpotent, where $p$ is the smallest prime dividing the order of $E$. Let $H$ be the Hall $p^{\prime}$-subgroup of $E$. If $H$ is non-trivial, then $G / H$ satisfies the hypothesis by Lemma 2.1 and consequently $G / H$ is supersoluble. By induction again, we have that $G$ is supersoluble. Hence one can assume that $E$ is a $p$-group and $E$ is non-cyclic. Let $G^{\mathfrak{U}}$ denote the smallest normal subgroup of $G$ such that $G / G^{\mathfrak{U}}$ is supersoluble. If $G^{\mathfrak{U}}<E$, then $G$ is supersoluble by Lemma 2.1 and induction. Hence we suppose that $E=G^{\mathfrak{U}}$.

We assert that $G$ is supersoluble. If not, then $G$ is a minimal non-supersoluble group by Lemma 2.1. Therefore, $G$ has the following properties: (1) $E / \Phi(E)$ is a non-cyclic chief factor of $G$; (2) $\exp (E)=p$ or 4 (if $p=2$ ). If $\exp (E)=p$, then every chief factor of $G$ below $E$ is cyclic by Lemma 4.2 and so $G$ is supersoluble, a contradiction. This shows that $p=2$ and $\exp (P)=4$. Pick $x \in E \backslash \Phi(E)$ such that $|x|=4$. Set $L=\langle x\rangle$ and $R=\Phi(E)$. First suppose that $L$ has a supersoluble supplement $T$ in $G$. Then $E / R \cap T R / R$ is normal in $G / R$ and so $E / R \cap T R / R=1$ or $E / R$. If $E / R \cap T R / R=1$, then $E=L$, which means that $E$ is cyclic, a contradiction. If $E / R \cap T R / R=E / R$, then $G=T$ is supersoluble, a contradiction. Hence $L$ has no supersoluble supplement in $G$ and so it is weakly $\Phi$-supplemented in $G$ by the hypothesis. Then there exists a subgroup $T$ of $G$ such that $G=L T$ and $L \cap T \leq \Phi(L)=\left\langle x^{2}\right\rangle$. If $E / R \cap T R / R=1$, then $E$ is cyclic as above, a contradiction. If $E / R \cap T R / R=E / R$, then $G=T$ and so $L \cap T=L$, contradicting that $L \cap T \leq\left\langle x^{2}\right\rangle$. Hence $G$ is supersoluble. 
Theorem 4.4 A group $G$ is supersoluble if and only if $G$ has a normal subgroup $E$ such that $G / E$ is supersoluble, and for each non-cyclic Sylow subgroup $P$ of $F^{*}(E)$, every cyclic subgroup of $P$ of order prime or 4 (if $P$ is a non-abelian 2-group) without any supersoluble supplement in $G$ is weakly $\Phi$-supplemented in $G$.

Proof The necessity is obvious and we only need to prove the sufficiency. Suppose this is not true and let $G$ be a counterexample of with $|G|+|E|$ minimal. We will derive a contradiction through the following steps.

(1) $F=F^{*}(E)=F(E)<E$.

Let $F=F^{*}(E)$. Then $F$ is soluble by Lemmas 2.1 and 4.1. Therefore $F=F^{*}(E)=F(E)$ by Lemma 2.2. If $F=E$, then $G$ is supersoluble by Theorem 4.3. This contradiction implies that $F<E$.

(2) Let $p$ be the smallest prime dividing the order of $F$. Then $p$ is odd.

Suppose that $p=2$. Let $P$ be a Sylow 2-subgroup of $F$, and let $Q$ be an arbitrary Sylow $q$-subgroup of $E$, where $q$ is an odd prime. Then $P$ is normal in $G$ and $P Q$ is 2-nilpotent by the hypothesis, Lemmas 2.1 and 4.1. Hence $Q \leq C_{E}(P)$ and so $O^{2}(E) \leq C_{E}(P)$. Set $V / P=$ $F^{*}(E / P)$ and $W=O^{2}(V) P$. Then $W$ is a normal subgroup of $G$. Since $O^{2}(E) \leq C_{E}(P)$, it is immediate that every chief factor of $W$ below $P$ is central in $W$. It follows that $W$ is quasinilpotent and so $W \leq F^{*}(E)=F(E)$. This shows that $W$ is nilpotent and therefore $V$ is soluble. Thus, $V / P=F^{*}(E / P)$ is nilpotent. Again, since $O^{2}(E) \leq C_{E}(P)$, we see that $V$ is nilpotent. Therefore $V=F(E)=F^{*}(E)$ and so $F^{*}(E / P)=F^{*}(E) / P=F(E) / P$. By Lemma 2.1 and the choice of $G, G / P$ satisfies the hypothesis and therefore is supersoluble. It follows from Theorem 4.3 that $G$ is supersoluble, a contradiction. Hence $p$ is an odd prime.

Now, let $P$ be the Sylow $p$-subgroup of $F$.

(3) Let $D$ be a normal subgroup of $G$ contained in $P$ such that every chief factor of $G$ below $D$ is cyclic. Suppose that $1=D_{0} \leq D_{1} \leq \cdots \leq D_{t}=D$ is a chief series of $G$ below $D$ and $C=\bigcap_{i=1}^{t} C_{i}$, where $C_{i}=C_{G}\left(D_{i} / D_{i-1}\right)$. Then $E \leq C$.

It is easy to see that $G / C$ is abelian. Since $F^{*}(E)=F(E) \leq E \cap C, F^{*}(E \cap C)=F^{*}(E)$. If $E \cap C \neq E$, then the pair $(G, E \cap C)$ satisfies the hypothesis and $|G|+|E \cap C|$ is less that $|G|+|E|$. Thus, $G$ is supersoluble by Lemma 2.1 and the choice of $G$. Hence, $E \leq C$, as desired.

(4) $P$ is non-cyclic.

Suppose that $P$ is cyclic. Then $E$ stabilizes a chain of subgroups of $P$ by (3), which implies that $E / C_{E}(P)$ is a $p$-group. Hence $O^{p}(E) \leq C_{E}(P)$. Arguing as in (2), we conclude that $G / P$ satisfies the hypothesis and therefore is supersoluble by the choice of $G$. In view of Theorem 4.3, $G$ is supersoluble, contrary to the choice of $G$.

Final contradiction.

By (4), $P$ is non-cyclic. Since $p$ is an odd prime by (2), $P$ contains a characteristic subgroup $D$ of exponent $p$ such that every non-trivial $p^{\prime}$-automorphism of $P$ induces a nontrivial automorphism of $D$. By Lemma 4.2, every chief factor of $G$ below $D$ is cyclic. Hence, by (3), $E / C_{E}(D)$ is a $p$-group, which implies that $E / C_{E}(P)$ is still a $p$-group by the property of $D$. Hence $O^{p}(E) \leq C_{E}(P)$. Analogously to the discussion in (2), one can deduce that $G / P$ satisfies the hypothesis and consequently $G / P$ is supersoluble. Now, by Theorem $4.3, G$ is supersoluble, a final violation finishing the proof. 


\section{Competing interests}

The authors declare that they have no competing interests.

\section{Authors' contributions}

$J$ carried out the new characterizations of supersolubility. FX conceived of the study and carried out the new characterizations of nilpotency. All authors read and approved the final manuscript.

\section{Author details}

${ }^{1}$ Department of Mathematics, Chongqing University of Arts and Science, Chongqing, 402160, P.R. China. ${ }^{2}$ Mathematics and Information Engineering Department, Humanistic Management College of Anyang Normal University, Anyang, 455000, P.R. China.

\section{Acknowledgements}

The authors are grateful to the referees for their helpful suggestions. This work was supported by the National Natural Science Foundation of China (Grant Nos. 11271301, 11171364, 11001226), the Scientific Research Foundation of Chongqing Municipal Education Committee (Grant No. KJ131204), the Science Fund for Creative Research Groups of Chongqing (Grant No. KJTD201321) and the Scientific Research Foundation of Chongqing University of Arts and Sciences (Grant Nos. R2012SC21, Z2012SC25)

\section{Received: 12 April 2013 Accepted: 22 August 2013 Published: 10 September 2013}

\section{References}

1. Arad, Z, Ward, MB: New criteria for the solubility of finite groups. J. Algebra 77, 234-246 (1982)

2. Ballester-Bolinches, A, Guo, XY: On complemented subgroups of finite groups. Arch. Math. 72, 161-166 (1999)

3. Hall, P: A characteristic property of soluble groups. J. Lond. Math. Soc. 12, 188-200 (1937)

4. Miao, L: On complemented subgroups of finite groups. Czechoslov. Math. J. 56, 1019-1028 (2006)

5. Guo, WB: The Theory of Classes of Groups. Kluwer Academic, Dordrecht (2000)

6. Huppert, B, Blackburn, N: Finite Groups III. Springer, Berlin (1982)

7. Ballester-Bolinches, A, Ezquerro, LM, Skiba, AN: Subgroups of finite groups with a strong cover-avoidance property. Bull. Aust. Math. Soc. 79, 499-506 (2009)

8. Huppert, B: Endliche Gruppen I. Springer, New York (1967)

\section{Submit your manuscript to a SpringerOpen ${ }^{\circ}$ journal and benefit from:}

- Convenient online submission

- Rigorous peer review

- Immediate publication on acceptance

Open access: articles freely available online

- High visibility within the field

- Retaining the copyright to your article 